\title{
Hypervitaminosis A Following the Ingestion of Fish Liver: Report on 3 Cases from the Poison Control Center in Marseille
}

\author{
Corinne Schmitt, PharmD ${ }^{1}$; Bastien Domangé, $\mathrm{MD}^{1}$; Romain Torrents, $\mathrm{MD}, \mathrm{PhD}^{1,2}$; \\ Luc de Haro, $\mathrm{MD}, \mathrm{PhD}^{1}$; Nicolas Simon, $\mathrm{MD}, \mathrm{PhD}^{1,2}$ \\ ${ }^{1}$ Clinical Pharmacology, Poison Control Center, St Marguerite Hospital, Marseille, France; ${ }^{2}$ Aix-Marseille Univ, APHM, INSERM, IRD, SESSTIM, St \\ Marguerite Hospital, Clinical Pharmacology, Poison control Center, Marseille, France
}

\begin{abstract}
In European countries, vitamin A toxicity is most often the result of an excessive intake of vitamin supplements and rarely the consequence of the ingestion of a large carnivorous fish liver. We report 3 cases of vitamin $\mathrm{A}$ poisoning after fish liver ingestion in mainland and overseas France. The patients were a 12-y-old girl, a 36-y-old pregnant woman, and a 62-y-old man. They experienced headache, nausea, emesis, and desquamation. Laboratory examination showed a high serum retinol level in the girl. The woman's pregnancy progressed to a miscarriage. This case series shows that this kind of poisoning is not restricted to the polar regions. In patients presenting with flushing combined with signs of intracranial hypertension, accurate questioning of the patient's diet is crucial to avoid misdiagnosis and unnecessary examinations. Pregnant women or women of child-bearing age should be informed of the risk to pregnancy in the case of excessive fish liver ingestion.
\end{abstract}

Keywords: acute fish liver poisoning, vitamin A poisoning, scombroid poisoning

\section{Introduction}

Vitamin A, also known as retinol, is a lipid-soluble vitamin that is available only through animal food sources. It is concentrated in the liver as retinyl esters and can reach very high levels in carnivores at the top of the food chain. ${ }^{1-3}$ Thus, the ingestion of liver from large fish or carnivorous vertebrates leads to a risk of toxicity.

In the early 20th century, Arctic explorers experienced adverse effects in humans and dogs after the consumption of the livers of polar bears, whales, and seals. Native inhabitants of the polar regions are aware of this risk and are careful about the amounts of liver they eat from the polar bears or seals they hunt. ${ }^{4-6}$ In some Asian cultures, fish liver is eaten frequently, and cases of poisoning in these regions have been reported within the last few years after ingestion of red snapper, grouper, seerfish, or wreckfish. ${ }^{5,7-11}$

Corresponding author: Corinne Schmitt, PharmD, Poison Control Center in Marseille, St Marguerite Hospital Marseille, France; e-mail: corinne.schmitt@ap-hm.fr.

Submitted for publication December 2019.

Accepted for publication June 2020.
Fish liver is also eaten in mainland and overseas France, sometimes leading to toxicity, as is illustrated in these cases attended through telephone calls by toxicologists at the poison control center in Marseille (PCCM). All of these cases took place within the area under the PCCM's responsibility (ie, Southeast France and French territories in the Indian Ocean).

\section{Case 1}

In 2014, in the southeast of France, a 62-y-old man with a medical history of hypercholesterolemia treated with atorvastatin caught a tuna weighing approximately $75 \mathrm{~kg}$. He fried its liver, which weighed about $1 \mathrm{~kg}$, and shared it with 2 guests. Two hours after the meal, all 3 people developed nausea, hepatalgia, facial flushing, and severe headaches. The patient went to the emergency department the following morning due to persistence of these symptoms. First, he was thought to have scombroid poisoning and was given antihistamine treatment. However, the PCCM, consulted within the following hours, suggested a diagnosis of hypervitaminosis A due to a large ingestion of fish liver. 
The patient was given analgesic treatment. Standard blood test results were unremarkable, and retinol levels were not measured. On Day 2, the headaches and nausea regressed but the flushing persisted and skin desquamation began. He was discharged home. Finally, the patient's flushing regressed, but skin desquamation was progressive from his scalp (Day 3) to his legs (on Day 19) via his shoulders and arms. The other 2 guests who shared the liver with him also had desquamation, whereas other guests who had only eaten tuna meat remained asymptomatic.

\section{Case 2}

In 2019, the PCCM was consulted by the pediatric emergency department on Reunion Island regarding a 12-y-old girl with major digestive symptoms combined with a severe headache that began a few hours after having shared the liver of a $200 \mathrm{~kg}$ marlin (Istiophoridae family) with her family. This patient, with a medical history of asthma and a strabismus, had an evening meal with her parents and her 18-y-old sister during which they shared the liver of a fish cooked by her mother. She woke the following morning with a frontal and periorbital headache with eye pain and phonophobia and a flushed face; she felt nauseated and vomited approximately 10 times. Her parents also experienced mild headaches with eye pain and flushing. Her older sister only experienced headaches.

When our patient arrived at the emergency department, she was weak, the vomiting had stopped, and no signs of dehydration or digestive disorders were observed. Her headache was diffuse with no photophobia. She was treated with metoclopramide, fluids by infusion, and progressive oral feeding. Serum ionogram, blood count, and blood gas analysis were unremarkable. A blood sample collected 18 $\mathrm{h}$ postingestion was sent to mainland France to evaluate retinol levels. Results were obtained within $10 \mathrm{~d}$, showing a retinol level of $1458 \mathrm{mg} \cdot \mathrm{L}^{-1}$ (normal 444-945), (or $5.09 \mathrm{mmol} \cdot \mathrm{L}^{-1}$ [normal $\left.1.55-3.30\right]$ ). She left the emergency department $36 \mathrm{~h}$ postingestion with the beginnings of superficial perioral desquamation. When she arrived back home, her strabismus worsened, leading her to consult an ophthalmologist. The headaches lasted for a few more days and were relieved with paracetamol. She also had myalgia with a favorable outcome. Two months after ingestion, the patient had still desquamation on the soles of her feet, but her strabismus had returned to normal. The whole family also experienced desquamation from Day 2 postingestion with favorable outcome within 2 mo.

\section{Case 3}

Later in 2019, the PCCM was contacted by a 36-y-old woman with a medical history of hypothyroidism treated with levothyroxine. She had eaten approximately $100 \mathrm{~g}$ of monkfish and cod liver 4 times over a period of $15 \mathrm{~d}$. Eight days after the last meal, she developed headaches, nausea, diarrhea, and diffuse arthralgia. This was when she discovered that she was pregnant (3 wk of gestation). Retinol blood levels at Day 10 after the last meal were normal. She followed the PCCM's advice and made an appointment at the gynecology department, but the pregnancy progressed to a miscarriage prior to the eighth week of gestation, before the patient saw the gynecologist.

\section{Discussion}

Vitamin A toxicity is mainly the result of an excessive intake of vitamin supplements. ${ }^{1}$ In Europe, it is rarely the consequence of the ingestion of a large carnivorous fish liver; however, our case series shows that this may occur. Recommendations are published on vitamin intakes and the upper levels not to be exceeded. According to the European food safety authority ${ }^{12}$ the recommended daily intake for retinol is $750 \mu \mathrm{g}$ for men, $650 \mu \mathrm{g}$ for women, and $600 \mu \mathrm{g}$ for children between 11 and $14 \mathrm{y}$ old. Signs of severe poisoning appear at 100 times the recommended daily dose in adults or 20 times in children. ${ }^{1,2}$ One gram of fish liver usually contains between 100 and $150 \mu \mathrm{g}$ of vitamin $\mathrm{A},{ }^{2}$ but levels measured in the Bahamas and in Taiwan in liver from different species of fish were much higher, reaching up to 5000 $\mu \mathrm{g} \cdot \mathrm{g}^{-1}$ liver. ${ }^{4,8,10}$ A 12 -y-old child would only need to eat $3 \mathrm{~g}$ of liver to be poisoned.

The teratogenic effect of an excess of retinol has been shown in several studies; however, the threshold of toxicity remains under debate. ${ }^{2,13}$ According to the research center for teratogenic agents in Paris, ${ }^{3}$ there is a risk of birth defect with doses above $3000 \mu \mathrm{g} \cdot \mathrm{d}^{-1}$ during the first term of pregnancy. This dose was greatly exceeded 4 times for Patient 3.

In adults and children, hypervitaminosis A has a typical toxidrome with the appearance of nausea, vomiting, flushing, vision disorders, myalgia, headaches, and even intracranial hypertension a few hours after ingestion. Over the following days, skin and mucosal desquamation appears and can last for several weeks. Some patients have vesicles or blisters and even hair loss..$^{4,7,9,10}$ Biological disorders such as hypercalcemia, high levels of hepatic enzymes, increased prothrombin time, and anemia have been reported. ${ }^{1,11}$ Treatment is mainly supportive using analgesics, fluids by infusion, and medical monitoring for signs of raised intracranial pressure because functional neurological prognosis may be at stake., ${ }^{5,6,9}$

Anamnesis, the clinical picture, and the collective nature of food poisoning leaves little doubt as to the diagnosis of the first 2 cases. Furthermore, the blood test carried out for Patient 2 confirmed the excessive level of retinol. In the third case, we do not know whether the excessive 
vitamin intake worsened an already established risk of early pregnancy loss (36-y-old; hypothyroidism).

Five cases of hypervitaminosis A have already been reported to the PCCM after ingestion of tuna, amberjack, and grouper liver but with no biological confirmation. ${ }^{5,14}$ The few cases of poisoning from fish liver with available retinol blood levels were consistent with the results of Patient 2 (between 4.94 and $6.20 \mu \mathrm{mol} \cdot \mathrm{L}^{-1}$ ). ${ }^{6,10,11} \mathrm{How}$ ever, blood retinol levels are not the best markers for vitamin A levels. Indeed, 75 to $80 \%$ of retinol is stored in the liver, and the concentration of retinol in the bloodstream is regulated by multiple mechanisms, including the synthesis of retinoic acid and its metabolites. ${ }^{1}$ Ideally, it would be best to measure retinyl ester levels in a liver biopsy, but this is not possible in standard clinical practice. ${ }^{2}$ Levels were measured in a biopsy from a 25 -y-old woman who had eaten shark liver, resulting in $18 \mathrm{mg}$ per $100 \mathrm{~g}$ liver tissue. $^{6}$

In addition to vitamin A, liver from deep-sea fish may contain substantial amounts of vitamin $\mathrm{D}$, with a possibility of vitamin D excess and thus hypercalcemia after ingestion. ${ }^{10}$

In some cases, hypervitaminosis A is mistaken for the most frequent type of fish poisoning in Europe: scombroid poisoning. This is genuine histamine poisoning that occurs after ingestion of meat from improperly stored Scombroidea fish. This underlines the importance of collecting proper anamnesis from the patient. ${ }^{9,14}$ Indeed, this toxidrome, combining flushing, rashes, headaches, nausea, and vomiting after ingestion of improperly stored fish, could be diagnosed by error; however, scombroid poisoning symptoms appear rapidly after ingestion, regress with antihistamine treatment, and do not progress to desquamation.

This small case series is a description of records issued from one PCC to understand more about this rare type of poisoning. Inherent limitations are the same as in every case report. This report has no epidemiological value; all the more so, as cases of hypervitaminosis A are probably underreported. Currently, PCCM records are only derived from spontaneous phone calls from patients or physicians, and some cases were probably mistaken for another type of fish poisoning.

\section{Conclusions}

These cases reported in mainland and overseas France confirm that hypervitaminosis A after ingestion of fish liver is not limited to the polar regions. High degree of suspicion, proper medical history, and physical examination are important to avoid misdiagnosis of vitamin A toxicity in patients with a history of fish consumption. Pregnant women or women of child-bearing age should be informed of the risk to pregnancy in the case of excessive ingestion of carnivorous fish liver.

Author Contributions: Study concept and design (CS, BD, NS, LDH); acquisition of the data (CS, BD, RT); data analysis (CS RT); drafting of the manuscript (CS, LDH). All authors read and approved the manuscript. Financial/Material Support: None.

Disclosures: None.

\section{References}

1. Penniston KL, Tanumihardjo SA. The acute and chronic toxic effects of vitamin A. Am J Clin Nutr. 2006;83(2):191-201.

2. Vidailhet M, Rieu D, Feillet F, Bocquet A, Chouraqui JP, Darmaun D, et al. Vitamin A in pediatrics: an update from the Nutrition Committee of the French Society of Pediatrics. Arch Pediatr. 2017;24(3):288-97.

3. Elefant E, Jardel A. In: IXème Symposium de la Société francophone Vitamines et biofacteurs. Ann Biol Chem. 2008;66:350-7.

4. Dewailly E, Rouja P, Schultz E, Julien P, Tucker T. Vitamin A intoxication from reef fish liver consumption in Bermuda. J Food Prot. 2011;74(9):1581-3.

5. Ardigier G, Drouet G, de Haro L. Poisoning after ingestion of Mediterranean greater amberjack liver: hypervitaminosis A clinical feature. Presse Med. 2010;39(7-8):833-4.

6. Misbah SA, Peiris JB, Atukorala TM. Ingestion of shark liver associated with pseudotumor cerebri due to acute hypervitaminosis A. J Neurol Neurosurg Psychiatry. 1984;47(2):216.

7. Chiu YK, Lai MS, Ho JC, Chen JB. Acute fish liver intoxication: report of three cases. Changgeng Yi Xue Za Zhi. 1999;22(3):468-73.

8. Hwang DF, Lu CH, Lin WF. Species identification and vitamin A level in lutjanid fish implicated in vitamin A poisoning. J Food Prot. 2010;73(4):769-73.

9. Hayman RM, Dalziel SR. Acute vitamin A toxicity: a report of three paediatric cases. J Paediatr Child Health. 2012;48(3):E98-100.

10. Chang $\mathrm{CH}$, Lu CW, Chung WH, Ho HC. Acute fish liver intoxication induced blisters formation and generalized skin peeling. Clin Toxicol (Phila). 2018;56(2):146-8.

11. Homma Y, Otani N, Ishimatsu S. A case report of acute vitamin A intoxication due to ocean perch liver ingestion. J Emerg Med. 2015;49(1):15-7.

12. EFSA Panel on Dietetic Products, Nutrition and Allergies. Scientific opinion on dietary reference values for vitamin A. EFSA Journal. 2015;13(3):4028.

13. Hathcock JN, Hattan DG, Jenkins MY, McDonald JT, Sundaresan PR, Wilkening VL. Evaluation of vitamin A toxicity. Am J Clin Nutr. 1990;52(2):183-202.

14. de Haro L, Prost N, Arditti J, David JM, Jouglard J. Scombroid poisoning. Experience of the anti-poison center of Marseille. Presse Med. 1997;26(27):1294. 